\title{
Antenna Selection in Spatial Modulation Systems
}

\author{
Rakshith Rajashekar, Student Member, IEEE, K. V. S. Hari, Senior Member, IEEE, \\ and L. Hanzo, Fellow, IEEE
}

\begin{abstract}
Novel transmit antenna selection techniques are conceived for Spatial Modulation (SM) systems and their symbol error rate (SER) performance is investigated. Specifically, low-complexity Euclidean Distance optimized Antenna Selection (EDAS) and Capacity Optimized Antenna Selection (COAS) are studied. It is observed that the COAS scheme gives a better SER performance than the EDAS scheme. We show that the proposed antenna selection based SM systems are capable of attaining a significant gain in signal-to-noise ratio (SNR) compared to conventional SM systems, and also outperform the conventional MIMO systems employing antenna selection at both low and medium SNRs.
\end{abstract}

Index Terms-Spatial modulation, antenna selection, diversity, limited feedback, capacity.

\section{PRELIMINARIES}

W HEN considering a Spatial Modulation (SM) system [1]-[4] having $N_{r}$ receive and $N_{t}$ transmit antennas, which relies on a single RF chain at the transmitter, we have the system model of $\mathbf{y}=\sqrt{\rho} \mathbf{h}_{i} s+\mathbf{n}$, where $\mathbf{y} \in \mathbb{C}^{N_{r}}$ is the received signal vector, $\rho$ is the average Signal-to-Noise Ratio (SNR) at each receive antenna, $s$ is a random symbol selected from a unit-energy $M$-QAM or -PSK signal set represented by $S, \mathbf{h}_{i}$ is the channel vector corresponding to the $i^{\text {th }}$ transmit antenna, and $\mathbf{n} \in \mathbb{C}^{N_{r}}$ is the noise vector. The entries of both $\mathbf{n}$ and of the channel matrix $\mathbf{H}$ obey the circularly symmetric complex-valued Gaussian distribution $\mathcal{C N}(0,1)$. In $\mathrm{SM}$, the input bitstream is divided into blocks of $\log _{2}\left(N_{t} M\right)$ bits and in each such block, $\log _{2} M$ bits select a symbol $s$ from an $M$-QAM or $M$-PSK signal set, while $\log _{2} N_{t}$ bits select an antenna $i$ out of $N_{t}$ transmit antennas for the transmission of the selected symbol $s$. Therefore, an SM symbol is comprised of the transmit antenna index and of the transmitted symbol from a conventional signal set. Let $L=\{i\}_{i=1}^{N_{t}}$ represent the set of transmit antenna indices. Assuming perfect Channel State Information at the Receiver (CSIR), the Maximum Likelihood (ML) detector conceived for this SM scheme is given by $(\hat{i}, \hat{s})_{M L}=\arg \min _{i \in L, s \in S}\left\|\mathbf{y}-\sqrt{\rho} \mathbf{h}_{i} s\right\|_{2}^{2}$.

Let $\mathcal{A}$ represent the event of an antenna index error and $\mathcal{S}$ represent the event of a transmitted symbol error under ML detection. Then, the probability of a SM symbol error is given

Manuscript received November 25, 2012. The associate editor coordinating the review of this letter and approving it for publication was Z. Hadzi-Velkov.

R. Rajashekar and K. V. S. Hari are with the Department of Electrical Communication Engineering, Indian Institute of Science, India (e-mail: \{rakshithmr, hari\}@ece.iisc.ernet.in).

L. Hanzo is with the School of ECS, University of Southampton, UK (email: lh@ecs.soton.ac.uk).

The financial support of the DST, India and of the EPSRC, UK under the auspices of the India-UK Advanced Technology Center (IU-ATC) as well as that of the European Research Council's Adavanced Fellow Grant is gratefully acknowledged.

Digital Object Identifier 10.1109/LCOMM.2013.012213.122650 by $P_{e}(S M)=\operatorname{Pr}(\mathcal{A})+\operatorname{Pr}\left(\mathcal{S}, \mathcal{A}^{c}\right)$, where $\mathcal{A}^{c}$ represents the complement of $\mathcal{A}$. Bounds on $\operatorname{Pr}(\mathcal{A})$ and $\operatorname{Pr}\left(\mathcal{S}, \mathcal{A}^{c}\right)$ can be easily derived, and are given by

$$
\begin{aligned}
\operatorname{Pr}(\mathcal{A}) & \leq \frac{1}{2 M^{2} N_{t}^{2}} \sum_{i=1}^{N_{t}} \sum_{\substack{j=1 \\
j \neq i}}^{N_{t}} \sum_{s \in S} \sum_{s^{\prime} \in S} \mathrm{E}\left[\exp \left(\frac{-\rho}{4}\left\|\mathbf{h}_{i} s-\mathbf{h}_{j} s^{\prime}\right\|^{2}\right)\right] \\
& \approx \frac{1}{2 M^{2}} \sum_{s \in S} \sum_{s^{\prime} \in S} \frac{1}{\left(1+\frac{\rho}{4}\left\{|s|^{2}+\left|s^{\prime}\right|^{2}\right\}\right)^{N_{r}}}
\end{aligned}
$$

and

$$
\begin{aligned}
\operatorname{Pr}\left(\mathcal{S}, \mathcal{A}^{c}\right) & \leq \frac{1}{2 M^{2} N_{t}} \sum_{i=1}^{N_{t}} \sum_{s \in S} \sum_{\substack{s^{\prime} \in S \\
s \neq s^{\prime}}} \mathrm{E}\left[\exp \left(\frac{-\rho}{4}\left\|\mathbf{h}_{i}\right\|^{2}\left|s-s^{\prime}\right|^{2}\right)\right] \\
& \approx \frac{1}{2 M^{2}} \sum_{s \in S} \sum_{\substack{s \in S \\
s \neq s^{\prime}}} \frac{1}{\left(1+\frac{\rho}{4}\left|s-s^{\prime}\right|^{2}\right)^{N_{r}}} .
\end{aligned}
$$

It is clear from (2) and (4) ${ }^{1}$ that the diversity order of both $\operatorname{Pr}(\mathcal{A})$ and $\operatorname{Pr}\left(\mathcal{S}, \mathcal{A}^{c}\right)$ is only $N_{r}$ and hence the diversity order of $P_{e}(S M)$ is $N_{r}$. Fig. 1 plots $P_{e}(S M), \operatorname{Pr}(\mathcal{A})$ and $\operatorname{Pr}\left(\mathcal{S}, \mathcal{A}^{c}\right)$ explicitly, considering an SM system having $N_{t}=4$ and $N_{r}=$ 2 for various throughputs. Two important observations may be inferred from these plots:

1) The diversity order (slope of the SER curve), associated with $\operatorname{Pr}(\mathcal{A})$ and $\operatorname{Pr}\left(\mathcal{S}, \mathcal{A}^{c}\right)$ are the same as formulated in (2) and (4).

2) As the number of bits/symbol increases (size of the QAM constellation), $P_{e}(S M)$ is dominated by the probability $\operatorname{Pr}\left(\mathcal{S}, \mathcal{A}^{c}\right)$.

Observe that $\operatorname{Pr}\left(\mathcal{S}, \mathcal{A}^{c}\right)$ given in (4) is same as the union bound on the error probability of a single-input multipleoutput system, whose diversity order can be increased by employing transmit antenna selection [6]-[8]. Our main idea in this paper is to exploit this fact to improve the performance of the SM system by employing transmit antenna selection.

Recently, an adaptive modulation order based SM scheme was introduced in [9], while combined modulation order and transmit antenna selection was conceived for SM in [10]. The minimum Euclidean distance among the legitimate transmit vectors was chosen as the decision metric for selecting the transmission mode in both [9] and [10]. However, the SER performance of these schemes [9], [10] was benchmarked only in comparison to the conventional SM scheme. Against this background, the new contributions of this paper are:

1) We propose a reduced-complexity Euclidean distance based transmit antenna selection scheme for the SM system.

2) We introduce a novel capacity-optimized transmit antenna selection scheme, which is capable of outperforming the Eu-

\footnotetext{
${ }^{1}$ Note that the sum of the bounds given in (2) and (4) gives union bound on the SM sysbol error probability.
} 

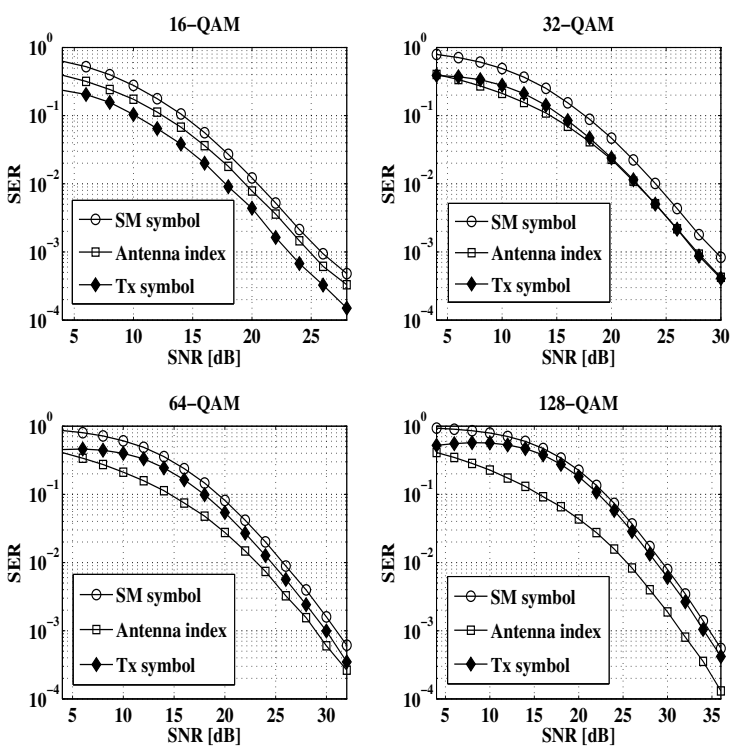

Fig. 1. Symbol Error Rate (SER) performance in a conventional SM system having $N_{t}=4, N_{r}=2$ and employing 16,32, 64, and 128-QAM to achieve $6,7,8$ and 9 bits per channel use (bpcu), respectively. In each plot, the error rates of the effective SM symbol $\left[P_{e}(S M)\right]$, Antenna index $[\operatorname{Pr}(\mathcal{A})]$ and the transmitted symbol $\left[\operatorname{Pr}\left(\mathcal{S}, \mathcal{A}^{c}\right)\right]$ are explicitly given. In all our simulation results presented in this paper, we have assumed frequency-flat block Rayleigh fading channel.

clidean distance based selection scheme - despite its reduced complexity.

3) The SER performance of both the proposed schemes is compared to that of the conventional SM and of the MIMO system employing transmit antenna selection.

\section{Proposed Selection Schemes For SM System}

\section{A. Euclidean Distance optimized Antenna Selection (EDAS)}

Let $\mathcal{I}$ represent the set of enumerations of all possible $n=$ $\left(\begin{array}{c}N_{t} \\ N_{S M}\end{array}\right)$ combinations of selecting $N_{S M}$ out of $N_{t}$ transmit antennas. Among the $\left(\begin{array}{c}N_{t} \\ N_{S M}\end{array}\right)$ possibilities, the specific antenna set that maximizes the minimum Euclidean distance among all possible transmit vectors [10] is obtained as

$$
I_{E D}=\arg \max _{I \in \mathcal{I}}\left\{\min _{\mathbf{x}_{1} \neq \mathbf{x}_{2} \in \chi}\left\|\mathbf{H}_{I}\left(\mathbf{x}_{1}-\mathbf{x}_{2}\right)\right\|_{2}^{2}\right\},
$$

where $\mathbf{H}_{I} \in \mathbb{C}^{N_{r} \times N_{S M}}$ has $N_{S M}$ columns given by $I$ and $\chi$ represents the set of all possible transmit vectors given by $\left\{\mathbf{e}_{i} s\right\}_{i=1}^{N_{S M}}$, where $s \in S$ and $\mathbf{e}_{i}$ is a $N_{S M} \times 1$ vector having 1 as the only non-zero element at the $i^{\text {th }}$ location, with $|\chi|=$ $N_{S M} M$. Further details on EDAS can be found in [10]. It may be readily seen from (5) that the order ${ }^{2}$ of complexity imposed by computing $I_{E D}$ is $\mathcal{O}\left(n N_{S M}^{2} M^{2}\right)$, which is significant. We will show that the complexity order for this selection scheme can be reduced to $\mathcal{O}\left(N_{t}^{2} M\right)$, when the signal set employed is a separable QAM set ${ }^{3}$.

\footnotetext{
${ }^{2}$ The order of complexity of an optimization problem is defined as the number of times the optimization metric is evaluated. If $\mathcal{C}$ is the computational complexity in evaluating the metric once, then the overall computational complexity is given by [order of complexity $\times \mathcal{C}$ ]. Thus, minimizing the order minimizes the overall computational complexity.

${ }^{3}$ A QAM signal set is said to be separable if it can be written as a Cartesian product of two PAM constellations.
}

1) Proposed low-complexity EDAS: We exploit the InterAntenna Interference (IAI)-free property of the SM system and the separability of the QAM signal sets for the sake of reducing the order of complexity in computing $I_{E D}$ of (5).

Step 1: Let us consider an upper triangular matrix $\mathbf{D} \in \mathbb{R}^{N_{t} \times N_{t}}$ whose $(i, j)^{\text {th }}$ entry $\mathbf{D}_{i, j}$ for $i>j$ is given by $\min _{s_{1}, s_{2} \in S}\left\|\mathbf{h}_{i} s_{1}-\mathbf{h}_{j} s_{2}\right\|_{2}^{2}$ and for $i=j$ we have $\min _{s_{1} \neq s_{2} \in S}\left\|\mathbf{h}_{i}\right\|_{2}^{2}\left|s_{1}-s_{2}\right|^{2}$. The computation of $I_{E D}$ in (5) may be equivalently written in terms of $\mathbf{D}$ as

$$
I_{E D}=\arg \max _{I \in \mathcal{I}}\{\min \mathbf{D}(I)\},
$$

where $\mathbf{D}(I)$ is a $\left(N_{S M} \times N_{S M}\right)$-element upper triangular sub-matrix of $\mathbf{D}$ obtained by deleting the specific rows and columns of $\mathbf{D}$ that are not in $I$ and $\{\min \mathbf{D}(I)\}$ represents the minimum of the non-zero elements of the matrix $\mathbf{D}(I)$. Thus, the order of complexity reduces to that of computing D. Note that $\mathbf{D}$ has $\frac{N_{t}\left(N_{t}-1\right)}{2}$ elements and the complexity involved in computing each element is $\mathcal{O}\left(M^{2}\right)$.

Step 2: The $(i, j)^{\text {th }}$ element of $\mathbf{D}$ for $i \neq j$ is given by

$$
\mathbf{D}_{i, j}=\min _{s_{1} \neq s_{2} \in S}\left\|\mathbf{h}_{i} s_{1}-\mathbf{h}_{j} s_{2}\right\|_{2}^{2}=\min _{s_{1} \neq s_{2} \in S}\left\|\left[\mathbf{h}_{i} \mathbf{h}_{j}\right]\left[\begin{array}{c}
s_{1} \\
-s_{2}
\end{array}\right]\right\|_{2}^{2} \text {. }
$$

Expressing (7) in terms of the real-valued I and $\mathrm{Q}$ counterparts and taking the QR decomposition of the resultant channel matrix, we arrive at $\mathbf{D}_{i, j}=\min \begin{gathered}s_{1 I}, s_{2 I} \in N_{1}-P A M \\ s_{1 Q}, s_{2 Q} \in N_{2}-P A M\end{gathered}\left\|\mathbf{R}\left[\begin{array}{c}s_{1 I} \\ s_{1 Q} \\ -s_{2 I} \\ -s_{2 Q}\end{array}\right]\right\|_{2}^{2}$, where $s_{i I}=\Re\left(s_{i}\right), s_{i Q}=\Im\left(s_{i}\right)$ for $i=1,2$ and $\mathbf{R}$ is the $(4 \times 4)$-element upper triangular matrix having zero entries at locations $(1,2)$ and $(3,4)$. Thus, upon conditioning $s_{2 I}$ and $s_{2 Q}, s_{1 I}$ and $s_{1 Q}$ can be obtained directly by hard-limiting as follows:

$$
\begin{aligned}
& \hat{s}_{1 I}=\min \left[\max \left(2 \text { rnd }\left[\frac{u_{1}+1}{2}\right]-1,-N_{1}+1\right), N_{1}-1\right], \\
& \hat{s}_{1 Q}=\min \left[\max \left(2 \text { rnd }\left[\frac{u_{2}+1}{2}\right]-1,-N_{2}+1\right), N_{2}-1\right],
\end{aligned}
$$

where $u_{i}=\left(-r_{i, 3} s_{2 I}+r_{i, 4} s_{2 Q}\right) / r_{i, i}$ for $i=1,2$ and $r_{i, j}$ represents the $(i, j)^{\text {th }}$ entry of $\mathbf{R}$. In (8) and (9), the expression of $2 \mathrm{rnd}^{4}\left[\frac{u_{i}+1}{2}\right]-1$ corresponds to an integer of the form $\hat{z}=2 z-1 \ni z \in \mathbb{Z}$, which is nearest to $u_{i}$ and $\min \left[\max \left\{\hat{z},-N_{i}+1\right\}, N_{i}-1\right]$ ensures that the chosen point is within the $N_{i}$-PAM constellation. Since $s_{2}$ can take $M=N_{1} N_{2}$ values, the order of complexity imposed by computing $\mathbf{D}_{i j}$ is $\mathcal{O}(M)$. Therefore, the order of complexity associated with computing $\mathbf{D}$, and hence of $I_{E D}$, is $\mathcal{O}\left(N_{t}^{2} M\right)$.

Fig. 2 compares the SER performance of the SM system employing EDAS with that of the conventional MIMO (CMIMO) system employing SNR-optimized transmit antenna selection and also with that of the conventional SM system. We have used $N_{t}=6$ and $N_{S M}=4$ out of $N_{t}=6$ antennas were selected based on the EDAS criterion. For a fair comparison, the C-MIMO system employing SNR optimized antenna selection is assumed to have $N_{t}=6, N_{r}=2$ and operating at the same rate as that of the SM system employing EDAS. It is clear from Fig. 2 that our EDAS based SM scheme

\footnotetext{
${ }^{4} r n d(\cdot)$ rounds any real number to its nearest integer.
} 

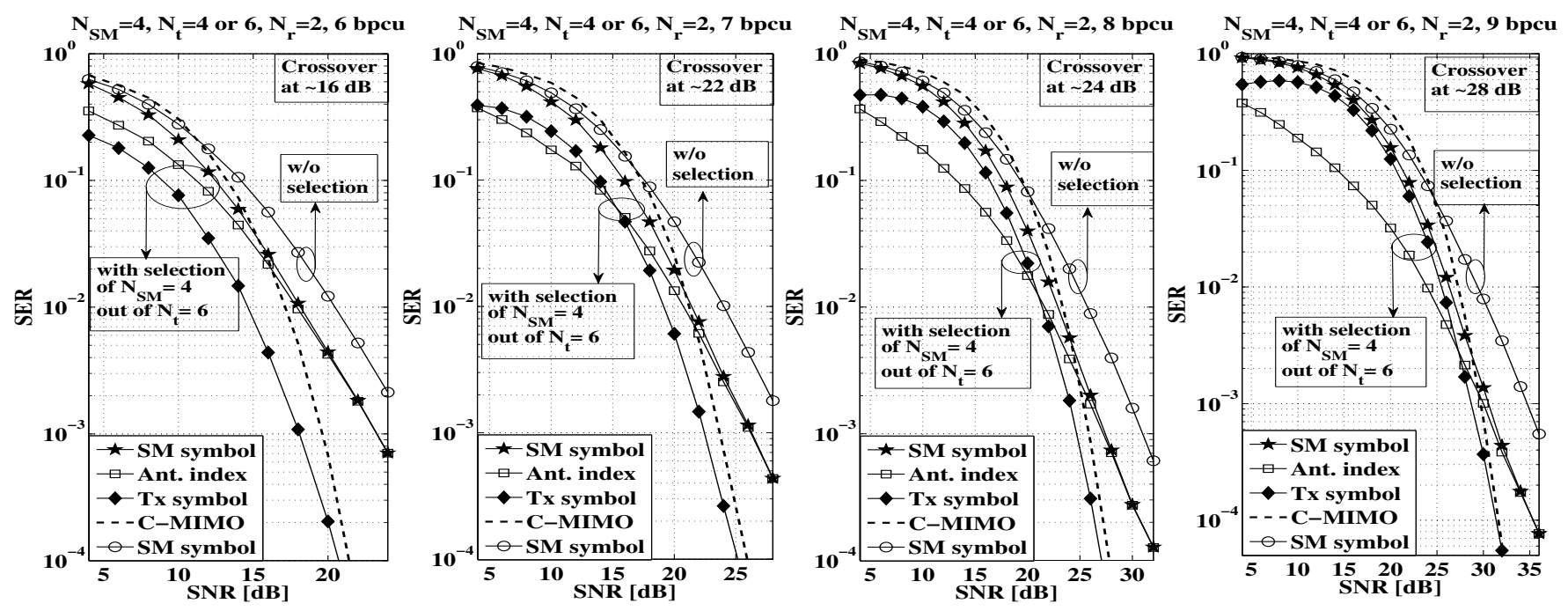

Fig. 2. Comparison of SER performance in an SM system employing EDAS, having $N_{t}=4$ or $6, N_{r}=2$ and employing 16-, 32-, 64- and 128-QAM to achieve 6, 7, 8 and $9 \mathrm{bpcu}$, respectively, to that of the conventional SM and MIMO system employing transmit antenna selection.

outperforms the C-MIMO employing antenna selection up to a certain SNR, which we term as the crossover point. Beyond the crossover point the C-MIMO system gives a better SER performance owing to its higher diversity order. Furthermore, it can be observed from Fig. 2 that as the data rate increases, the EDAS based selection scheme exhibits a higher crossover point. Table I lists the crossover points achieved for various rates as well as the SNR gain achieved with respect to the conventional SM system without antenna selection.

\section{B. Capacity Optimized Antenna Selection (COAS)}

For a given channel realization and SNR, the capacity of the SM system having $N_{S M}$ transmit antennas can be bounded as

$$
\alpha \leq C_{S M} \leq \alpha+\log _{2}\left(N_{S M}\right),
$$

where we have $\alpha=\frac{1}{N_{S M}} \sum_{i=1}^{N_{S M}} \log _{2}\left(1+\rho\left\|\mathbf{h}_{i}\right\|^{2}\right)$. It may be readily seen that $\alpha$ in (10) is maximized by choosing the $N_{S M}$ antennas corresponding to the largest channel norms out of the $N_{t}$ transmit antennas. The set of antenna indices corresponding to the $N_{S M}$ largest channel norms may be computed at the receiver for every coherence interval and fed back to the transmitter. This set is given by $I_{C O}=\left\{i_{1}, i_{2}, \ldots, i_{N_{S M}}\right\}$, which expects that

$\left\|\mathbf{h}_{i_{1}}\right\|^{2}>\left\|\mathbf{h}_{i_{2}}\right\|^{2}>\cdots>\left\|\mathbf{h}_{i_{N_{S M}}}\right\|^{2}>\left\|\mathbf{h}_{i_{N_{S M}+1}}\right\|^{2}>\cdots>\left\|\mathbf{h}_{i_{N_{t}}}\right\|^{2}$.

Proposition 1: In an SM system employing COAS, the diversity order $d$ exhibited by the SER curve of $\left\{\mathcal{S} \mid \mathcal{A}^{c}\right\}$ is $\left(N_{t}-N_{S M}+1\right) N_{r}$.

Proof: The diversity order of the SER curve associated with $\left\{\mathcal{S} \mid \mathcal{A}^{c}\right\}$ is given by

$$
d=-\lim _{\rho \rightarrow \infty} \frac{\log \operatorname{Pr}\left(\mathcal{S}, \mathcal{A}^{c}\right)}{\log \rho} \triangleq-\lim _{\rho \rightarrow \infty} \frac{\log P_{\text {out }}\left(\mathcal{A}^{c}\right)}{\log \rho} .
$$

where, $P_{\text {out }}\left(\mathcal{A}^{c}\right)$ is the channel's outage probability, when there is no antenna index error. For a given channel realization, the capacity of the SM system employing COAS can be bounded as $C_{S M} \leq \frac{1}{N_{S M}} \sum_{i \in I_{C O}} \log _{2}\left(1+\rho\left\|\mathbf{h}_{i}\right\|^{2}\right)+\log _{2}\left(N_{S M}\right)$, where equality is achieved when there is no antenna index error. Assuming that indeed there is no antenna index error, an outage occurs, when $\log _{2}\left(1+\rho\left\|\mathbf{h}_{i_{N_{S M}}}\right\|^{2}\right)<R^{\prime}$, where $R^{\prime}=\log _{2}(M)$. This is plausible, since among the $N_{S M}$ largest-norm channel vectors, $\mathbf{h}_{i_{N_{S M}}}$ has the lowest norm. Thus, the outage probability in the presence of no antenna index error is given by $P_{\text {out }}\left(\mathcal{A}^{c}\right)=\operatorname{Pr}\left(\left\|\mathbf{h}_{i_{S M}}\right\|^{2}<\delta\right)$, where $\delta=\frac{2^{R^{\prime}}-1}{\rho}$. Note that we have

$$
\operatorname{Pr}\left(\left\|\mathbf{h}_{i_{N_{S M}}}\right\|^{2}<\delta\right)=\prod_{i=1}^{N_{t}-N_{S M}+1} \operatorname{Pr}\left(X_{i}<\delta\right),
$$

where $X_{i}$ are independent Chi-square distributed random variables (rv) having a pdf of $f(x)=\frac{1}{\left(N_{r}-1\right) !} x^{N_{r}-1} e^{-x}$, $x \geq 0$. Since at high SNRs we have $\operatorname{Pr}\left(X_{i}<\delta\right) \approx \frac{\delta^{N_{r}}}{N_{r} !}$ for $1 \leq i \leq N_{t}-N_{S M}+1$, (13) can be reduced to

$$
\operatorname{Pr}\left(\left\|\mathbf{h}_{i_{S M}}\right\|^{2}<\delta\right) \approx\left(\frac{\delta^{N_{r}}}{N_{r} !}\right)^{N_{t}-N_{S M}+1} .
$$

It may be readily inferred from (14) that $P_{\text {out }}\left(\mathcal{A}^{c}\right)$ decays as $\rho^{\left(N_{t}-N_{S M}+1\right) N_{r}}$, hence we will have $d=\left(N_{t}-N_{S M}+1\right) N_{r}$.

Note that the order of complexity in computing (11) is independent of the constellation size and grows only with $N_{t}$.

Fig. 3 compares the SER performance of the SM system employing COAS to that of the C-MIMO and the conventional SM system considered earlier. Similar to EDAS, the COAS also gives a better SER performance compared to the C-MIMO system employing antenna selection at low and medium SNRs. For SNRs beyond the crossover points, the C-MIMO system outperforms the COAS scheme due to its higher diversity order. Table I lists the crossover points of the COAS and also the SNR gain achieved with respect to the conventional SM system operating without antenna selection. It is readily seen from Table I that COAS gives a slightly better SNR gain and higher crossover points compared to EDAS. Furthermore, 
This article has been accepted for inclusion in a future issue of this journal. Content is final as presented, with the exception of pagination.

TABLE I

ACHIEVABLE SNR GaIN DUE TO PROPOSED ANTENNA SELECTION SCHEMES AT AN SER OF $10^{-3}$ WITH RESPECT TO A CONVENTIONAL SM SYSTEM HAVING $N_{S M}=N_{t}=4$ AND $N_{r}=2$, AND SER CROSSOVER POINTS ACHIEVED BY THEM WITH RESPECT TO C-MIMO SYSTEM EMPLOYING TRANSMIT ANTENNA SELECTION.

\begin{tabular}{|c||c|c||c|c|}
\hline $\begin{array}{c}\text { Rate } \\
\text { (bpcu) }\end{array}$ & $\begin{array}{c}\text { EDAS } \\
\text { SNR gain }[\mathrm{dB}]\end{array}$ & $\begin{array}{c}\text { COAS } \\
\text { SNR gain }[\mathrm{dB}]\end{array}$ & $\begin{array}{c}\text { EDAS crossover } \\
\text { point }[\mathrm{dB}]\end{array}$ & $\begin{array}{c}\text { COAS crossover } \\
\text { point }[\mathrm{dB}]\end{array}$ \\
\hline \hline 6 & 2.7 & 2.8 & 16 & 16 \\
\hline 7 & 3.5 & 3.6 & 21 & 22 \\
\hline 8 & 3.7 & 4.3 & 24 & 25 \\
\hline 9 & 4.4 & 5.3 & 28 & 30 \\
\hline
\end{tabular}
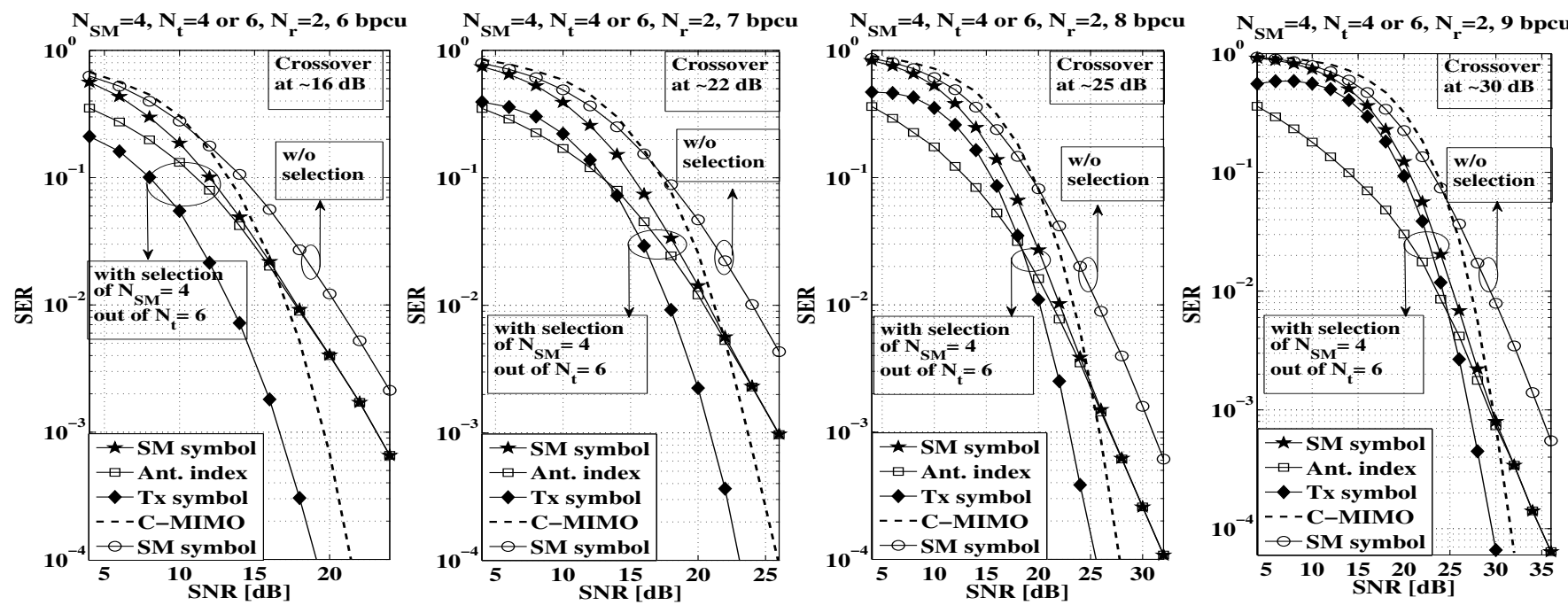

Fig. 3. Comparison of SER performance in an SM system employing COAS, having $N_{t}=4$ or $6, N_{r}=2$ and employing 16-, 32-, 64- and 128-QAM to achieve $6,7,8$ and $9 \mathrm{bpcu}$, respectively, to that of the conventional SM and MIMO system employing transmit antenna selection.

it is straightforward to infer from (6)-(9) and (11) that the complexity of implementing COAS is lower than that of EDAS. Thus, we conclude that the COAS scheme constitutes a beneficial choice for SM systems in terms of its performance versus complexity characteristics.

\section{CONCLUSiOnS}

Two novel antenna selection schemes, namely lowcomplexity EDAS and COAS were proposed in the paper. The SER performance of the proposed schemes is compared to both that of the SM system operating without antenna selection and to the conventional MIMO system employing transmit antenna selection. Our proposed schemes are observed to offer a significant SNR gain with respect to the conventional SM system and outperform the conventional MIMO system employing antenna selection at both low and medium SNRs. The performance of both these $N_{S M}$ out of $N_{t}$ antenna selection schemes was studied for various throughputs and the COAS was found to be a better choice due to its lower computational complexity and improved SER performance.

\section{REFERENCES}

[1] R. Mesleh, H. Haas, C. Ahn, and S. Yun "Spatial modulation-a new low complexity spectral efficiency enhancing technique," in Proc. 2006 International Conf. Commun. Netw., pp. 1-5.

[2] R. Mesleh, H. Haas, S. Sinanovic, C. Ahn, and S. Yun "Spatial modulation," IEEE Trans. Veh. Technol., vol. 57, no. 4, pp. 2228-2242, 2008.

[3] M. Di Renzo and H. Haas, "Performance comparison of different spatial modulation schemes in correlated fading channels," in Proc. 2010 IEEE International Conf. Commun.

[4] E. Basar, U. Aygolu, E. Panayirci, and H. V. Poor, "New trellis code design for spatial modulation," IEEE Trans. Wireless Commun., vol. 10, no. 9, pp. 2670-2680, Aug. 2011.

[5] E. Basar, U. Aygolu, E. Panayirci, and H. V. Poor, "Performance of spatial modulation in the presence of channel estimation errors," IEEE Commun. Lett., vol. 16, no. 2, pp. 176-179, Feb. 2012.

[6] S. Sanayei and A. Nosratinia, "Antenna selection in MIMO systems," IEEE Commun. Mag., vol. 42, no. 10, pp. 68-73, Oct. 2004.

[7] D. Love, R. Heath, V. Lau, D. Gesbert, B. Rao, and M. Andrews, "An overview of limited feedback in wireless communication systems," IEEE J. Sel. Areas Commun., vol. 26, no. 8, pp. 1341-1365, Oct. 2008.

[8] R. Heath and A. Paulraj, "Antenna selection for spatial multiplexing systems based on minimum error rate," in Proc. 2001 Int. Conf. Соттиnications, vol. 7, pp. 2276-2280.

[9] P. Yang, Y. Xiao, Y. Yu, and S. Li, "Adaptive spatial modulation for wireless MIMO transmission systems," IEEE Commun. Lett., vol. 15, no. 6, pp. 602-604, June 2011.

[10] P. Yang, Y. Xiao, L. Li, Q. Tang, Y. Yu, and S. Li, "Link adaptation for spatial modulation with limited feedback," IEEE Trans. Veh. Technol., vol. 61, no. 8, pp. 3808-3813, Oct. 2012. 